\title{
VERY EARLY AND EARLY ONSET SCHIZOPHRENIA SPECTRUM DISORDERS - DIAGNOSTIC CHALLENGE
}

\author{
Ilinca Untu ${ }^{1}$, Stefana Maria Moisa ${ }^{1}$, Stefan Lucian Burlea ${ }^{2}$, Anamaria Ciubara ${ }^{1}$, \\ Vasile Valeriu Lupu ${ }^{1}$, Dana-Teodora Anton-Paduraru ${ }^{1}$ \\ ${ }^{1}$ Psihiatrics Department, ,, Gr. T. Popa“, University of Medicine and Pharmacy, Iasi \\ ${ }^{3}$, , Gr. T. Popa " University of Medicine and Pharmacy, Iasi
}

\begin{abstract}
Very early onset and early onset schizophrenia - which manifest themselves before the age of 13 (the first) and 18 (the latter) - represent a little explored nosological entity, which entails a genuine diagnostic challenge, considering the specificities of young age. At the same time, childhood and adolescence onset schizophrenia intertwines with a series of neurocognitive development disorders, comprising an important genetic susceptibility. The entire context of its emergence, the difficulty of detecting psychoproductive symptoms during early childhood, the severity of symptoms and the chronicization impose a rigorous diagnostic behaviour and a multidisciplinary approach for optimal long-term therapy and for the socio-professional integration of patients. This paper aims at synthesizing the literature review, which may represent a starting point for ample future research; the purpose is to create specific guides on childhood and adolescence schizophrenia.
\end{abstract}

Keywords: early onset schizophrenia, very early onset schizophrenia, child and adolescent, neurocognitive development, genetic susceptibility

\section{INTRODUCTION}

Schizophrenia is a severe psychiatric disorder, characterized by thought disorganization and marked perceptual qualitative disturbances, accompanied by inadequate or flat affects, with significant impact on the patient's global functionality $(1,2)$.

Schizophreniain children or adolescents - also called early onset schizophrenia (onset before the age of 18) - is still little explored and known. The overlapping of this nosological entity and adult schizophrenia is extremely relative: certain clinical forms specific to childhood and adolescence must be carefully studied and detailed, for an optimal diagnostic and therapeutic approach $(1,2,3)$.

\section{EPIDEMIOLOGIC DATA}

The general prevalence of schizophrenia is approximately $1 \%$, but its incidence is far more significant, considering its chronic character. Though schizophrenia usually targets the adult population, an estimated $20 \%$ of patients with schizophrenia experience the onset before the age of $19(1,2)$.

The frequency of the onset increases with age. Hence, $1 \%$ of the onsets occur before the age of 10 , $4 \%$ before $15 ; 43 \%$ of males and $28 \%$ of females before the age of 19. Currently, it is posited that early onset schizophrenia debuts before 18 and very early onset schizophrenia debuts before 13 . Thus far, epidemiologic data on adolescent and especially on child schizophrenia is scarce, compared to the same adult pathology. The more homogeneous defining of diagnostic subgroups led to data that are more viable. However, the data collected so far is still insufficient: data reports a prevalence of very early and early onset schizophrenia of $0.03 \%$; less than a fifth of this percentage is represented by very early onset schizophrenia. It is imperious to discover the particularities of these forms of schizophrenia; more insight into this pathology may lead to the creation of specific therapeutic guides, ad- 
justed to the age and specificities of this population segment $(2,3,4)$.

\section{ELEMENTS OF ETIOLOGY}

Very early and early onset schizophrenia is considered a heterogeneous nosological category: both genetic and environmental factors may contribute to its debut. Its significant familial aggregation is already a certified notion; pre-morbid psychopathological elements are more severe and frequent in children/adolescents than in adults, as they are based on neuro-developmental and cytogenetic anomalies $(4,5,6)$.

Most pathological elements of a neurocognitive, linguistic and psychosocial nature, as well as alterations of brain structures are similar among adults and children suffering from schizophrenia. This suggests the existence of a common neuro-biological basis for adult onset schizophrenia and for very early/early onset schizophrenia $(4,6,7)$.

\section{PARTICULARITIES OF THE CLINICAL ASPECT IN VERY EARLY/EARLY ONSET SCHIZOPHRENIA}

Formally, very early and early onset schizophreniashares the same diagnostic criteria with adult schizophrenia. Nevertheless, it is worth mentioning several clinical-anamnestic particularities of early onset schizophrenia, grouped into three categories: the form with normal pre-morbid functioning and rapid alteration of mental structures, the evolving forms of autism spectrum disorder and the forms with suspicion of organicity $(2,3)$.

The developmental level of children/adolescents has a unique impact on the expression of various semiological aspects determined by very early and early onset schizophrenia. In this sense, we mention difficulties in outlining hallucinations and/ or delirious ideation, caused by insufficient cognitive development and language skills. Therefore, the diagnosing of this form of schizophrenia can prove a challenge $(2,6)$.

Systematized and detailed delirious ideation is rare among very young children. At the same time, thought disorganisation and hallucinations usually occur after the age of six; they increase in frequency after 8-9. Among pre-school children, it is fundamental to discern between fantastic ideas related to sleep or included within the development, products of imagination and psychotic phenomena. Moreover, children may experience transitory hallucinations, in the context of stressful situations or high anxiety. These elements make it even more difficult to diagnose very early onset schizophrenia $(2,6,8)$.

The complexity of psychotic phenomena increases with age and it is directly proportional with the level of development. Hence, in adolescence, psychotic symptomatology is similar to that of adults. Nonetheless, it is essential to have a correct differential diagnostic, which does not underestimate the existence of a distinct psychiatric condition - such as borderline disorder - when the clinician observes short-term psychotic episodes $(1,2,6)$.

Though scientific studies suggest that the intelligence of children and adolescents with very early and early onset schizophrenia ranges below the mean of general population $(10-20 \%$ of this patients have an IQ below 70), the limited number of individuals analyzed makes it highly difficult to draw a pertinent and well-defined conclusion (2).

\section{THE ISSUE OF DIFFERENTIAL DIAGNOSTIC}

The difficulty of setting a positive diagnostic of schizophrenia in children and adolescents is accentuated by the mandatory presence of a rigorous differential diagnostic. Hence, the clinician must take into account the existence of emotional psychosis (depression with psychotic symptoms or bipolar disorder, where the fundamental element of distinction is the flat affects, present within schizophrenia), of schizoaffective disorders (insufficiently researched among children/adolescents), of Asperger's and of other autism spectrum disorders (DSM V does not exclude schizophrenia within autism spectrum disorders, if psychoproductive phenomena last for at least one month), of disintegrative child psychosis, of neurodegenerative disorders, of psychotic phenomena caused by the ingestion of psychoactive substances and, not least, of temporal lobe epilepsy $(2,6,9)$.

\section{PARTICULARITIES OF THE FORM WITH PRE-MORBID FUNCTIONING AND GRADUAL ALTERATION (SCHIZOPHRENIA PER SE)}

The form with pre-morbid functioning and gradual alterationis the type ofearly onset schizophrenia most similar to adult schizophrenia. Its general clinical characteristics are not fundamentally different from adult schizophrenia, in terms of diagnostic criteria determined according to ICD-10 and DSM V. Differences between these forms of schizophrenia are quantitative more than qualitative. In fact, though the symptomatology per se is identical, 
studies have found that the earlier the onset, the more dominant negative symptomatology. Habitually, the onset precedes clinical evidence: it is insidious, accompanied by various degrees of egodystonia (the patient recognizes to a certain extent the abnormality of his/her experiences and he/she tends to share them with the adults). Most often, adults show anguish and destabilization, and children or adolescents become reticent in sharing symptomatology with adults, psychiatrists included; however, the patient is partially aware of its pathologic character (that is highly different from adult schizophrenia, where the patient is completely unaware of his/her condition). The almost pathognomonic aspect of adult schizophreniacharacterized by behavioural bizarreness and serious difficulties in establishing relationships - is rarely present in early onset schizophrenia, partly due to ego-dystonia. This clinical particularity may lead to diagnostic errors: often, these symptoms are mistaken for depression; it is an essential element of the differential diagnostic, in relation to aboulia, social withdrawal, loss of facial expressiveness, though sad mood is rarely present $(2,9,10)$.

On the other hand, beyond the manifested symptomatology of the patient, it was proven that the earlier the onset, the more frequent pathological antecedents associated during developmental stages. The insidious onset of the condition is more frequent than brutal onsets (lacking anticipatory signs). At the same time, it is important to underscore that numerous patients with adult onset schizophrenia present pre-morbid psychopathological antecedents dating from childhood $(8,9,10)$.

A series of studies underline that in early onset schizophrenia, often purely psychotic symptoms are preceded by an alteration of language, cognition and behaviour. In many cases, pre-morbid antecedents include minimal neurodevelopment disturbances, attention and emotional deficit, as well as discrete language disorders. Sometimes, these patients are marked by lateralization and motor coordination disorders $(11,12,13)$.

Early onset schizophrenia associates with increased severity, significant hereditary influence and a predisposition of the male gender (common element of many early onset psychiatric disorders) (4).

This classic and consensual form of schizophrenia that affects individuals younger than 18 is distinguished by a dominant negative symptomatology, characterized by more varied and numerous pre-morbid symptoms than adult onset schizophrenia (2).

\section{SCHIZOPHRENIA AS EVOLVING FORM OF AUTISM SPECTRUM DISORDER}

Schizophrenia as evolving form of autism spectrum disorder does not represent a consensual version; actually, it is still a subject of controversy. In the context of severe communication disorders and frequent association of psycho-cognitive development delay, the diagnostic of psychoproductive phenomena (hallucinations and delirious ideation) is extremely difficult. Diagnosing schizophrenia can become a challenge. Some studies suggest that certain forms of autism - such as non-specific disorders of the spectrum and Asperger's syndrome can evolve to schizophrenia. Therefore, autism may constitute a risk factor for schizophrenia $(2,10)$.

From a clinical perspective, it features the symptomatology characteristic to schizophrenia, though the general aspect is marked by negative signs: hallucinations and delirious ideation are harder to pinpoint $(2,3)$.

\section{FORMS OF SCHIZOPHRENIA WITH ASPECT OF ORGANICITY}

The association of schizophrenia spectrum disorder and organic pathologies - some of them very rare - is not unusual. Moreover, in case of genetic diseases, there is a genuine entanglement with the neurodevelopment and psychoactive development planes. These forms of schizophrenia feature a series of atypical elements, which suggest the existence of an organic pathology associated with schizophreniform symptomatology. The atypical characteris evoked in case of first rank signs (mostly visual hallucinations, confusing elements, catatonic elements and resistance to classic antipsychotic medication) and second rank signs (acute or very early onset, pathological neurodevelopment, psycho-cognitive development delay, family history of psychosis) $(9,13)$.

\section{THERAPEUTIC COORDINATES}

The entire therapeutic behaviour must focus on the chronic character of this pathology; therefore, continuity in the multidisciplinary care of the patients must be ensured $(9,14)$.

From the perspective of medication, the first choice is atypical antipsychotics (olanzapine, risperidone, aripiprazole, amisulpride); most of them have been approved for children older than 13 . Data within scientific literature - still scarce - suggests that the frequency of adverse effects of anti- 
psychotics (dyskinesia, weight gain and sedation are significant) is higher among them than among adults. If a 12-week treatment fails to be effective, it is recommended to associatethe antipsychotics. If the association of two antipsychotics fails to work after another 12 weeks, the patients suffers from resistant schizophrenia and the advice is to consider using clozapine (however, it is essential to monitor full blood count, considering the risk of agranulocytosis). Irrespective of the antipsychotic administered as long-term treatment, it is fundamental to monitor the potential emergence of adverse effects, such as metabolic syndrome, dyskinesia/dystonia, excessive sedation (most of the times transitory) and, not least, decrease in libido in case of adolescents $(14,15)$.

\section{CONCLUSIONS}

Early onset schizophrenias are particular forms of schizophrenia and they should benefit from a clinical-therapeutic and ab initio approach; such an approach must be diagnostic, correct and efficient. Genetic and somatic evaluation is fundamental in all cases.

The improvement of long-term prognostic is crucial; it can be achieved by creating social and educational support systems for these children/adolescents. At the same time, psychotherapy for both the patient and his/her family leads to better integration of the schizophrenic individual in the sociofamilial environment.

\section{REFERENCES}

1. Kinros J., Reichenberg A., Frangou S. The neurodevelopmental theory of schizophrenia: evidence from studies of early onset cases. The Israel journal of psychiatry and related sciences, 2010; 47(2):110-7

2. Bonnot O., Cohen D. Schizophrénie à début précoce. In: Ferrari P., Bonnot O. (ed). Traité européen de psychiatrie et psychopatologie de l'enfant et de l'adolescent. Paris: Lavoisier-Médecine Sciences, 2012

3. Kyriakopoulos M., Frangou S. Pathophysiology of early onset schizophrenia. Int Rev Psychiatry. 2007; 19:315-324.

4. Gejman P.V., Sanders A.R., Duan J. The role of genetics in the etiology of schizophrenia. Psychiatr Clin North Am. 2010; 33:35-66.

5. National Alliance on Mental Illness. Early onset schizophrenia. [cited 2013 July 12]. Available from: http://www.nami.org/Content/ ContentGroups/Helpline1/Early_Onset_Sc hizophrenia.htm.

6. Kumra S., Charles Schulz S. Editorial: Research progress in early-onset schizophrenia. Schizophr Bull. 2008; 34:15-17.

7. Thomas G., Genest P., Walter M. Can concepts of neurodevelopment and neuroplasticity explain post-traumatic psychoses? Neuro-Chirurgie, 2009 Feb; 55(1):36-9

8. Nicolson R., Lenane M., Singaracharlu S. Premorbid speech and language impairments in childhood-onset schizophrenia: association with risk factors. The American journal of psychiatry, 2000 May; 157(5):794-800
9. Eggers C. Schizophrenia in childhood and adolescence. Symptomatology, clinical course, etiological and therapeutic aspects. Zeitschrift fur arztliche Fortbildung und Qualitatssicherung, 2002 Oct; 96(9):567-77

10. Gilmore J.H. Understanding what causes schizophrenia: a developmental perspective. The American journal of psychiatry, 2010 Jan; 167(1):8-10

11. Eggers C., Bunk D., Early development of childhood-onset schizophrenia. Fortschritte der Neurologie-Psychiatrie, 2009 Oct; 77(10):558-67

12. Walterfang M., Bonnot O., Mocellin R. The neuropsychiatry of inborn errors of metabolism. J Inherit Metab Dis, 2013; 36:687-702

13. Bonnot O., Cohen D. Trouble psychiatriques et cognitifs associes aux maladies neurométabolique. Rev Neurol 2011; 167:881-885

14. Lahutte B., Cornic F., Multidisciplinary approach of organic catatonia in children and adolescents may improve treatment decision making. Prog Neuropsychopharmacol Biol Psychiatry, 2008; 32:1393-1398

15. Bennot O., Consoli A., Cohen D., Les antipsychotiques chez l'enfant et adolescent. Psychiatrie EM- Cd, editor. Paris:Elsevier, 2010 